\title{
New Results on Passivity Analysis for Uncertain Neural Networks with Time-Varying Delay
}

\author{
Xueying Shao, ${ }^{1}$ Qing Lu, ${ }^{2}$ Hamid Reza Karimi, ${ }^{3}$ and Jin $\mathrm{Zhu}^{1}$ \\ ${ }^{1}$ School of Mathematics and Physics, Bohai University, Jinzhou, Liaoning 121013, China \\ ${ }^{2}$ College of Engineering, Bohai University, Jinzhou, Liaoning 121013, China \\ ${ }^{3}$ Department of Engineering of the Faculty of Engineering and Science, University of Agder, 4898 Grimstad, Norway
}

Correspondence should be addressed to Qing Lu; luqing.bella@gmail.com

Received 26 January 2014; Accepted 12 February 2014; Published 24 March 2014

Academic Editor: Ming Liu

Copyright (C) 2014 Xueying Shao et al. This is an open access article distributed under the Creative Commons Attribution License, which permits unrestricted use, distribution, and reproduction in any medium, provided the original work is properly cited.

\begin{abstract}
The paper investigates the stability and passivity analysis problems for a class of uncertain neural networks with time-delay via delta operator approach. Both the parameter uncertainty and the generalized activation functions are considered in this paper. By constructing an appropriate Lyapunov-Krasovskii functional, some new stability and passivity conditions are obtained in terms of linear matrix inequalities (LMIs). The main characteristic of this paper is to obtain novel stability and passivity analysis criteria for uncertain neural networks with time-delay in the delta operator system framework. A numerical example is presented to demonstrate the effectiveness of the proposed results.
\end{abstract}

\section{Introduction}

Recently, neural networks have attracted considerable attention due to their applications in wide areas such as associative memory $[1,2]$, pattern recognition $[1,3]$, and optimization problems [4-7]. Recently, some stability conditions [8-12] and passivity analysis for neural networks [13-15] have been reported in the literature. The effect of time-delays [16-25] cannot be ignored in the real systems due to the facts that the delays can lead to instability [26-29], oscillation, or chaos. Recently, the stability results of time-delay neural networks have been presented in [30-36]. In addition, many results on passivity of neural networks with time-delay have been proposed [37-41].

It is well known that the discrete systems are often used for computer realization and continuous systems are frequently applied to theoretical analysis, respectively. Sampling continuous systems can lead to considerable discrete systems. When the sampling is fast using the traditional shift operator, the poles are located in the stable boundary. Then, the discrete systems will lose stability in finite word length computer. Goodwin proposed delta operator approach in [42] which is used to replace the aforementioned operator with sample continuous systems, which can unify some previous related results of the continuous and discrete systems into the framework of the delta operator systems. The delta operator is defined by

$$
\delta x(t)= \begin{cases}\frac{d x(t)}{d t}, & T=0 ; \\ \frac{x(t+T)-x(t)}{T}, & T \neq 0,\end{cases}
$$

where $T$ is a sampling period. More recently, much attention has been focused on the stability and stabilization problems for some delta operator systems [43-47]. However, it should be mentioned that there are few achievements about passivity analysis for uncertain discrete neural networks with timevarying delay via delta operator approach; instability exists in the applications of neural networks when the sampling rate is high, which motivates this research.

In this paper, the stability and passivity problems are investigated for uncertain neural networks with time-varying delay via delta operator approach. Both the parameter uncertainty and the generalized activation functions are considered in this paper. By choosing a new type of Lyapunov functional in delta domain and employing some novel methods to handle the delays, some stability and passivity criteria are 
proposed. The proposed conditions are expressed in terms of linear matrix inequalities (LMIs), which are dependent on the sampling period. The main characteristic of this paper is to obtain some stability and passivity analysis criteria for uncertain neural networks with time-varying delay in the delta operator system framework. Finally, a numerical example is given to demonstrate the effectiveness of the developed results.

Notation. Throughout this paper, for the sake of convenience, we use $t_{k}$ to denote $k T$, where $T$ is the sampling period. $\mathbb{R}^{n}$ denotes the $n$-dimensional Euclidean space. The superscript $T$ stands for matrix transposition. The notation $\operatorname{diag}\{\cdots\}$ denotes a block-diagonal matrix. For real symmetric matrices $X$ and $Y$, the notation $X \geq Y$ (resp., $X>Y$ ) means that the $X-Y$ is positive semidefinite (resp., positive-definite). $I$ is the identity matrix with appropriate dimensions. The symbol “*” stands for the symmetric term in a matrix. Matrices, if their dimensions are not explicitly stated, are assumed to have compatible dimensions.

\section{Problem Formulation}

Consider the following uncertain neural network with timevarying delay:

$$
\begin{aligned}
\delta x\left(t_{k}\right)= & -\left(W_{1}+\Delta W_{1}\left(t_{k}\right)\right) x\left(t_{k}\right) \\
& +\left(W_{2}+\Delta W_{2}\left(t_{k}\right)\right) f\left(x\left(t_{k}\right)\right) \\
& +\left(W_{3}+\Delta W_{3}\left(t_{k}\right)\right) f\left(x\left(t_{k}-d_{k}\right)\right) \\
& +u\left(t_{k}\right), \\
y\left(t_{k}\right)= & f\left(x\left(t_{k}\right)\right),
\end{aligned}
$$

where $x\left(t_{k}\right) \in \mathbb{R}^{n}$ stands for the network state at time $t_{k}$; $f\left(x\left(t_{k}\right)\right) \in \mathbb{R}^{n}$ denotes the activation at time $t_{k} ; u\left(t_{k}\right) \in \mathbb{R}^{n}$ is the external input at time $t_{k} ; y\left(t_{k}\right)$ is the output vector; $W_{1}$ is a positive diagonal matrix; $\Delta W_{1}\left(t_{k}\right), \Delta W_{2}\left(t_{k}\right)$, and $\Delta W_{3}\left(t_{k}\right)$ are unknown matrices; $d_{k}$ is a time-varying delay $0 \leq d_{m} \leq$ $d_{k} \leq d_{M}$, with $d_{m}=n_{m} T$ and $d_{M}=n_{M} T ; n_{m}$ and $n_{M}$ are two known positive and finite integers; $T$ is the sampling period. The time-varying parameter uncertainties $\Delta W_{1}\left(t_{k}\right), \Delta W_{2}\left(t_{k}\right)$, and $\Delta W_{3}\left(t_{k}\right)$ are assumed to be in the following form:

$$
\begin{aligned}
& {\left[\begin{array}{lll}
\Delta W_{1}\left(t_{k}\right) & \Delta W_{2}\left(t_{k}\right) & \Delta W_{3}\left(t_{k}\right)
\end{array}\right]} \\
& =H F\left(t_{k}\right)\left[\begin{array}{lll}
E_{1} & E_{2} & E_{3}
\end{array}\right],
\end{aligned}
$$

where $H, E_{1}, E_{2}$, and $E_{3}$ are known constant matrices; $F\left(t_{k}\right)$ is an unknown time-varying matrix satisfying

$$
F^{T}\left(t_{k}\right) F\left(t_{k}\right) \leq I \text {. }
$$

The activation function $f\left(x\left(t_{k}\right)\right)$ satisfies the following condition:

$$
K_{i}^{-} \leq \frac{f_{i}\left(\alpha_{1}\right)-f_{i}\left(\alpha_{2}\right)}{\alpha_{1}-\alpha_{2}} \leq K_{i}^{+}, \quad i=1,2, \ldots, n,
$$

with $\alpha_{1} \neq \alpha_{2}$,

$$
\begin{aligned}
& K^{-}=\operatorname{diag}\left[\begin{array}{llll}
K_{1}^{-} & K_{2}^{-} & \cdots & K_{n}^{-}
\end{array}\right], \\
& K^{+}=\operatorname{diag}\left[\begin{array}{llll}
K_{1}^{+} & K_{2}^{+} & \cdots & K_{n}^{+}
\end{array}\right] .
\end{aligned}
$$

Before ending this section, some preliminaries are recalled which are used to prove the main results in the next section.

Definition 1 (see [48]). A delta operator system is asymptotically stable, if the following conditions hold:

(i) $V(x(t)) \geq 0$ with equality if and only if $x(t)=0$;

(ii) $\delta V(x(t))=[V(x(t+T))-V(x(t))] / T<0$,

where $V(x(t))$ is a Lyapunov function in the delta domain.

Definition 2. The neural network (2) and (3) is called passive if there exists a scalar $\gamma \geq 0$ such that

$$
\sum_{k=0}^{\infty}\left[-\gamma u^{T}\left(t_{k}\right) u\left(t_{k}\right)-2 y^{T}\left(t_{k}\right) u\left(t_{k}\right)\right] \leq 0 .
$$

Lemma 3 (see [49]). The property of delta operator for any time function $x(t)$ and $y(t)$ can be represented as

$\delta(x(t) y(t))=\delta(x(t)) y(t)+x(t) \delta y(t)+T \delta(x(t)) \delta y(t)$,

where $T$ is a sampling period.

Lemma 4 (see [50]). For any constant positive semidefinite symmetric matrix $W$, two positive integers $r$ and $r_{0}$ satisfy $r \geq r_{0} \geq 1$; the following inequality holds:

$$
\begin{aligned}
& \left(\sum_{i=r_{0}}^{r} x(i)\right)^{T} W\left(\sum_{i=r_{0}}^{r} x(i)\right) \\
& \quad \leq\left(r-r_{0}+1\right) \sum_{i=r_{0}}^{r} x^{T}(i) W x(i) .
\end{aligned}
$$

Lemma 5 (see [51]). Let $U, V, W$, and $M$ be real matrices of appropriate dimensions with $M$ satisfying $M=M^{T}$; then $M+$ $U V W+W^{T} V^{T} U^{T}<0$ for all $V^{T} V \leq I$, if and only if there exists a scalar $\varepsilon>0$ such that $M+\varepsilon^{-1} U U^{T}+\varepsilon W^{T} W<0$.

Lemma 6 (see [52] Schur complement). Given constant matrices $\Omega_{1}, \Omega_{2}$, and $\Omega_{3}$ with appropriate dimensions, where $\Omega_{1}^{T}=\Omega_{1}$, and $\Omega_{2}^{T}=\Omega_{2}>0$, then $\Omega_{1}+\Omega_{3}^{T} \Omega_{2}^{-1} \Omega_{3}<0$, if and only if

$$
\left[\begin{array}{cc}
\Omega_{1} & \Omega_{3}^{T} \\
* & -\Omega_{2}
\end{array}\right]<0 \quad \text { or } \quad\left[\begin{array}{cc}
-\Omega_{2} & \Omega_{3} \\
* & \Omega_{1}
\end{array}\right]<0 .
$$

\section{Main Results}

In this section, the stability and the passivity results for discrete-time uncertain neural network with time-varying delay via delta operator are given. Firstly, the stability conditions are given in the following part. 
3.1. Stability Analysis. In order to consider the stability condition for uncertain neural networks with time-delay (2), we define $\Delta W_{i}\left(t_{k}\right)=0(i=1,2,3)$ and $u\left(t_{k}\right)=0$ in (2). Then, we can have the following neural networks with time-delay:

$$
\begin{aligned}
\delta x\left(t_{k}\right)= & -W_{1} x\left(t_{k}\right)+W_{2} f\left(x\left(t_{k}\right)\right) \\
& +W_{3} f\left(x\left(t_{k}-d_{k}\right)\right) .
\end{aligned}
$$

For neural networks with time-delay in (12), the stability criterion is obtained in the following theorem.

Theorem 7. For given scalars $0 \leq d_{m} \leq d_{k} \leq d_{M}$, neural network (12) with (6) is asymptotically stable, if there exist $P>$ $0, Q_{i}>0(i=1,2,3), R>0$, and $S>0$, positive definite diagonal matrices $L_{1}, L_{2}, X_{1}$, and $X_{2}$, such that the following LMI holds:

$$
\Xi=\left[\begin{array}{cc}
\Theta_{11} & \Theta_{12} \\
* & \Theta_{22}
\end{array}\right]<0
$$

where

$$
\begin{aligned}
& \Theta_{11}=\left[\begin{array}{ccc}
\Psi_{11} & -S W_{1} & 0 \\
* & \Psi_{22} & 0 \\
* & * & -Q_{2}
\end{array}\right], \\
& \Theta_{12}=\left[\begin{array}{ccc}
0 & S W_{2} & S W_{3} \\
\frac{1}{n_{M} T} R & P W_{2} & \Psi_{25} \\
0 & 0 & X_{2} K^{+}
\end{array}\right] \text {, } \\
& \Theta_{22}=\left[\begin{array}{ccc}
-Q_{1}-\frac{1}{n_{M} T} R & 0 & 0 \\
* & \Psi_{55} & 0 \\
* & * & \Psi_{66}
\end{array}\right] \text {, } \\
& \Psi_{11}=T P+T n_{M} R-S-S^{T}, \\
& \Psi_{22}=-2 L_{1} K^{-}+2 L_{2} K^{+}+Q_{1}+Q_{2} \\
& +T(d+1) Q_{2}-\frac{1}{n_{M} T} R \\
& -P W_{1}-W_{1}^{T} P \\
& \Psi_{25}=L_{1}-L_{2}+X_{1} K^{+}+P W_{2} \text {, } \\
& \Psi_{55}=-2 X_{1}+Q_{3} \text {, } \\
& \Psi_{66}=-2 X_{2}-Q_{3} \text {, } \\
& L_{1}=\operatorname{diag}\left\{\begin{array}{llll}
l_{11} & l_{12} & \cdots & l_{1 n}
\end{array}\right\}, \\
& L_{2}=\operatorname{diag}\left\{\begin{array}{llll}
l_{21} & l_{22} & \cdots & l_{2 n}
\end{array}\right\} \text {, } \\
& X_{1}=\operatorname{diag}\left\{\begin{array}{llll}
\lambda_{11} & \lambda_{12} & \cdots & \lambda_{1 n}
\end{array}\right\} \text {, } \\
& X_{2}=\operatorname{diag}\left\{\begin{array}{llll}
\lambda_{21} & \lambda_{22} & \cdots & \lambda_{2 n}
\end{array}\right\} \text {. }
\end{aligned}
$$

Proof. Choose a Lyapunov-Krasovskii functional in delta domain as follows:

$$
V\left(t_{k}\right)=\sum_{i=1}^{5} V_{i}\left(t_{k}\right),
$$

with

$$
\begin{aligned}
V_{1}\left(t_{k}\right)= & x^{T}\left(t_{k}\right) P x\left(t_{k}\right), \\
V_{2}\left(t_{k}\right)= & 2 T \sum_{j=1}^{n} l_{1 j} \int_{0}^{x_{j}\left(t_{k}-T\right)}\left(f_{j}(s)-K_{j}^{-} s\right) d s \\
& +2 T \sum_{j=1}^{n} l_{2 j} \int_{0}^{x_{j}\left(t_{k}-T\right)}\left(K_{j}^{+} s-f_{j}(s)\right) d s, \\
V_{3}\left(t_{k}\right)= & T \sum_{i=1}^{n_{M}} x^{T}\left(t_{k}-i T\right) Q_{1} x\left(t_{k}-i T\right) \\
& +T \sum_{i=1}^{n_{k}} x^{T}\left(t_{k}-i T\right) Q_{2} x\left(t_{k}-i T\right) \\
& +T \sum_{i=1}^{n_{k}} f^{T}\left(x\left(t_{k}-i T\right)\right) Q_{3} f\left(x\left(t_{k}-i T\right)\right), \\
V_{4}\left(t_{k}\right)= & T^{2} \sum_{i=n_{m}}^{n_{M}} \sum_{j=1}^{i} x^{T}\left(t_{k}-j T\right) Q_{3} x\left(t_{k}-j T\right), \\
V_{5}\left(t_{k}\right)= & \sum_{i=1}^{n_{M}} \sum_{j=1}^{i} e^{T}\left(t_{k}-j T\right) \operatorname{Re}\left(t_{k}-j T\right),
\end{aligned}
$$

where

$$
e\left(t_{k}\right)=x\left(t_{k}\right)-x\left(t_{k}+T\right)=-T \delta x\left(t_{k}\right) .
$$

Applying Lemma 3, the delta-domain form of $V_{1}\left(t_{k}\right)$ can be obtained as

$$
\begin{aligned}
\delta V_{1}\left(t_{k}\right)= & \delta^{T} x\left(t_{k}\right) P x\left(t_{k}\right)+x^{T}\left(t_{k}\right) P \delta x\left(t_{k}\right) \\
& +T \delta^{T} x\left(t_{k}\right) P \delta x\left(t_{k}\right) \\
= & -x^{T}\left(t_{k}\right) W_{1}^{T} P x\left(t_{k}\right)+x^{T}\left(t_{k}\right) W_{2}^{T} P f\left(x\left(t_{k}\right)\right) \\
& +x^{T}\left(t_{k}\right) W_{3}^{T} P f\left(x\left(t_{k}-d_{k}\right)\right) \\
& -x^{T}\left(t_{k}\right) P W_{1} x\left(t_{k}\right)+x^{T}\left(t_{k}\right) P W_{2} f\left(x\left(t_{k}\right)\right) \\
& +x^{T}\left(t_{k}\right) P W_{3} f\left(x\left(t_{k}-d_{k}\right)\right) \\
& +T \delta^{T} x\left(t_{k}\right) P \delta x\left(t_{k}\right) .
\end{aligned}
$$

From (6), for the scalars $\lambda_{1 i} \geq 0, \lambda_{2 i} \geq 0$, one can have

$$
\begin{aligned}
& 2 \sum_{i=1}^{n} \lambda_{1 i} f_{i}\left(x_{i}\left(t_{k}\right)\right)\left[f_{i}\left(x_{i}\left(t_{k}\right)\right)-K_{i}^{+} x_{i}\left(t_{k}\right) \leq 0\right], \\
& 2 \sum_{i=1}^{n} \lambda_{2 i} f_{i} x_{i}\left(t_{k}-n_{k} T\right) \\
& \quad \times\left[f_{i}\left(x_{i}\left(t_{k}-n_{k} T\right)\right)-K_{i}^{+} x_{i}\left(t_{k}-n_{k} T\right)\right] \leq 0,
\end{aligned}
$$


which can be equivalently denoted as

$$
\begin{aligned}
2 f^{T}( & \left.x\left(t_{k}\right)\right) X_{1} f\left(x\left(t_{k}\right)\right) \\
& -2 f^{T}\left(x\left(t_{k}\right)\right) K^{+} X_{1} x\left(t_{k}\right) \leq 0, \\
2 f^{T}\left(x\left(t_{k}-n_{k} T\right)\right) X_{2} f\left(x\left(t_{k}-n_{k} T\right)\right) & \\
& -2 f^{T}\left(x\left(t_{k}-n_{k} T\right)\right) K^{+} X_{2} x\left(t_{k}-n_{k} T\right) \leq 0 .
\end{aligned}
$$

Then, we obtain the following inequality:

$$
\begin{aligned}
\delta V_{2}\left(t_{k}\right)= & 2 \sum_{j=1}^{n} l_{1 j}\left[\int_{0}^{x_{j}\left(t_{k}\right)}\left(f_{j}(s)-K_{j}^{-} s\right) d s\right. \\
& \left.\quad-\int_{0}^{x_{j}\left(t_{k}-T\right)}\left(f_{j}(s)-K_{j}^{-} s\right) d s\right] \\
& +2 \sum_{j=1}^{n} l_{2 j}\left[\int_{0}^{x_{j}\left(t_{k}\right)}\left(K_{j}^{+} s-f_{j}(s)\right) d s\right. \\
& \\
\leq & 2 \sum_{j=1}^{n} l_{1 j} x_{j}\left(t_{k}\right)\left[f_{j}\left(x_{j}\left(t_{k}\right)\right)-K_{j}^{-} x\left(t_{k}\right)\right] \\
& +2 \sum_{j=1}^{n} l_{2 j} x_{j}\left(t_{k}\right)\left[K_{j}^{+} x\left(t_{k}\right)-f_{j}\left(x_{j}\left(t_{k}\right)\right)\right] \\
\leq & 2 x^{T}\left(t_{k}\right) L_{1}\left[f\left(x\left(t_{k}\right)\right)-K^{-} x\left(t_{k}\right)\right] \\
& +2 x^{T}\left(t_{k}\right) L_{2}\left[K^{+} x\left(t_{k}\right)-f\left(x\left(t_{k}\right)\right)\right] \\
& -2 f^{T}\left(x\left(t_{k}\right)\right) X_{1} f\left(x\left(t_{k}\right)\right) \\
& +2 f^{T}\left(x\left(t_{k}\right)\right) K^{+} X_{1} x\left(t_{k}\right) \\
& -2 f^{T}\left(x\left(t_{k}-n_{k} T\right)\right) X_{2} f\left(x\left(t_{k}-n_{k} T\right)\right) \\
& +2 f^{T}\left(x\left(t_{k}-n_{k} T\right)\right) K^{+} X_{2} x\left(t_{k}-n_{k} T\right) .
\end{aligned}
$$

Taking the delta operator manipulations of $V_{3}\left(t_{k}\right)$, we can obtain that

$$
\begin{aligned}
& \delta V_{3}\left(t_{k}\right) \\
& =\frac{1}{T} \times T\left[\sum_{i=1}^{n_{M}} x^{T}\left(t_{k}-i T+T\right) Q_{1} x\left(t_{k}-i T+T\right)\right. \\
& \left.-\sum_{i=1}^{n_{M}} x^{T}\left(t_{k}-i T\right) Q_{1} x\left(t_{k}-i T\right)\right] \\
& +\frac{1}{T} \times T\left[\sum_{i=1}^{n_{k}} x^{T}\left(t_{k}-i T+T\right) Q_{2} x\left(t_{k}-i T+T\right)\right. \\
& \left.-\sum_{i=1}^{n_{k}} x^{T}\left(t_{k}-i T\right) Q_{2} x\left(t_{k}-i T\right)\right] \\
& +\frac{1}{T} \times T\left[\sum_{i=1}^{n_{k}} f^{T}\left(x\left(t_{k}-i T+T\right)\right)\right. \\
& \times Q_{3} f\left(x\left(t_{k}-i T+T\right)\right) \\
& \left.-\sum_{i=1}^{n_{k}} f^{T}\left(x\left(t_{k}-i T\right)\right) Q_{3} f\left(x\left(t_{k}-i T\right)\right)\right]
\end{aligned}
$$

$$
\begin{aligned}
\leq & x^{T}\left(t_{k}\right) Q_{1} x\left(t_{k}\right)-x^{T}\left(t_{k}-n_{M} T\right) Q_{1} x\left(t_{k}-n_{M} T\right) \\
& +x^{T}\left(t_{k}\right) Q_{2} x\left(t_{k}\right)-x^{T}\left(t_{k}-n_{k} T\right) Q_{2} x\left(t_{k}-n_{k} T\right) \\
& +T \sum_{i=n_{m}}^{n_{M}} x^{T}\left(t_{k}-i T\right) Q_{2} x\left(t_{k}-i T\right) \\
& +f^{T}\left(x\left(t_{k}\right)\right) Q_{3} f\left(x\left(t_{k}\right)\right)-f^{T}\left(x\left(t_{k}-n_{k} T\right)\right) \\
& \times Q_{3} f\left(x\left(t_{k}-n_{k} T\right)\right) .
\end{aligned}
$$

Taking the delta operator manipulations of $V_{4}\left(t_{k}\right)$, the following results can be obtained:

$$
\begin{aligned}
& \delta V_{4}\left(t_{k}\right) \\
& =\frac{1}{T} \times T^{2}\left[\sum_{i=n_{m}}^{n_{M}} \sum_{j=1}^{i} x^{T}\left(t_{k}-j T+T\right)\right. \\
& \times Q_{3} x\left(t_{k}-j T+T\right) \\
& =T\left[\sum_{i=n_{m}}^{n_{M}} x^{T}\left(t_{k}\right) Q_{3} x\left(t_{k}\right)\right. \\
& \left.\times \sum_{j=1}^{n_{M}} x^{T}\left(t_{k}-j T\right) Q_{3} x\left(t_{k}-j T\right)\right] \\
& \left.=T(d+1) x^{T}\left(t_{k}-i T\right) Q_{3} x\left(t_{k}-i T\right)\right] \\
& -T \sum_{i=n_{m}}^{n_{M}} x^{T}\left(t_{k}-i T\right) Q_{3} x\left(t_{k}-i T\right),
\end{aligned}
$$

where $d=n_{M}-n_{m}$.

Taking the delta operator manipulations of $V_{5}\left(t_{k}\right)$ and using Lemma 4, it can be found that

$$
\begin{gathered}
\delta V_{5}\left(t_{k}\right) \\
=\frac{1}{T}\left[\sum_{i=1}^{n_{M}} \sum_{j=1}^{i} e^{T}\left(t_{k}-j T+T\right)\right. \\
\times \operatorname{Re}\left(t_{k}-j T+T\right) \\
\left.-\sum_{i=1}^{n_{M}} \sum_{j=1}^{i} e^{T}\left(t_{k}-j T\right) \operatorname{Re}\left(t_{k}-j T\right)\right] \\
=\frac{n_{M}}{T} e^{T}\left(t_{k}\right) \operatorname{Re}\left(t_{k}\right) \\
-\frac{1}{T} \sum_{i=1}^{n_{M}} e^{T}\left(t_{k}-i T\right) \operatorname{Re}\left(t_{k}-i T\right)
\end{gathered}
$$




$$
\begin{aligned}
\leq & T n_{M} \delta^{T} x\left(t_{k}\right) R \delta x\left(t_{k}\right) \\
& -\frac{1}{n_{M} T}\left[x\left(t_{k}-n_{M} T\right)-x\left(t_{k}\right)\right]^{T} \\
& \times R\left[x\left(t_{k}-n_{M} T\right)-x\left(t_{k}\right)\right] .
\end{aligned}
$$

For a given positive definite matrix $P_{1}$ with appropriately dimensions, one has that

$$
\begin{aligned}
r_{1}\left(t_{k}\right)=2 \delta^{T} x\left(t_{k}\right) S[ & -W_{1} x\left(t_{k}\right)+W_{2} f\left(x\left(t_{k}\right)\right) \\
& \left.+W_{3} f\left(x\left(t_{k}-d_{k}\right)\right)-\delta x\left(t_{k}\right)\right]=0 .
\end{aligned}
$$
holds:

Combining (18) and (21)-(26), the following inequality

$$
\begin{aligned}
\delta V\left(t_{k}\right) & =\sum_{i=1}^{5} \delta V_{i}\left(t_{k}\right) \\
& \leq \eta^{T}\left(x\left(t_{k}\right)\right) \Xi \eta\left(x\left(t_{k}\right)\right),
\end{aligned}
$$

where

$$
\begin{aligned}
\eta^{T}\left(x\left(t_{k}\right)\right)=[ & \delta^{T} x\left(t_{k}\right), x^{T}\left(t_{k}\right), x^{T}\left(t_{k}-n_{k} T\right), \\
& x^{T}\left(t_{k}-n_{M} T\right), f^{T}\left(x\left(t_{k}\right)\right), \\
& \left.f^{T}\left(x\left(t_{k}-n_{k} T\right)\right)\right] .
\end{aligned}
$$

It can be seen from Theorem 7 that $\Xi<0$, which means that $\delta V\left(t_{k}\right)<0$. Then, based on Definition 1 , neural network (12) is asymptotically stable. The proof is completed.

In the subsection, we continue to consider the robust stability problem of neural network (2) without inputs $u\left(t_{k}\right)$; equivalently, we have

$$
\begin{aligned}
\delta x\left(t_{k}\right)= & -\left(W_{1}+\Delta W_{1}\left(t_{k}\right)\right) x\left(t_{k}\right) \\
& +\left(W_{2}+\Delta W_{2}\left(t_{k}\right)\right) f\left(x\left(t_{k}\right)\right) \\
& +\left(W_{3}+\Delta W_{3}\left(t_{k}\right)\right) f\left(x\left(t_{k}-d_{k}\right)\right) .
\end{aligned}
$$

For neural network (29), the robust stability criterion is given in the following theorem

Theorem 8. For given scalars $0 \leq d_{m} \leq d_{k} \leq d_{M}$, neural network (29) under the conditions (4)-(6) is robustly asymptotically stable, if there exist $P>0, Q_{i}>0(i=1,2,3)$, $R>0, S>0$ positive definite diagonal matrices $L_{1}, L_{2} X_{1}$, and $X_{2}$, such that the following LMI holds:

$$
\widehat{\Xi}=\left[\begin{array}{cc}
\widehat{\Theta}_{11} & \widehat{\Theta}_{12} \\
* & \widehat{\Theta}_{22}
\end{array}\right]<0,
$$

where

$$
\begin{aligned}
& \widehat{\Theta}_{11}=\left[\begin{array}{cccc}
\Psi_{11} & S W_{1} & 0 & 0 \\
* & \Psi_{22} & 0 & \frac{1}{n_{M} T} R \\
* & * & -Q_{2} & 0 \\
* & * & * & -Q_{1}-\frac{1}{n_{M} T} R
\end{array}\right], \\
& \widehat{\Theta}_{12}=\left[\begin{array}{cccc}
S W_{2} & S W_{3} & S H & 0 \\
\Psi_{25} & P W_{3} & P H & -\varepsilon E_{1}^{T} \\
0 & X_{2} K^{+} & 0 & 0 \\
0 & 0 & 0 & 0
\end{array}\right], \\
& \widehat{\Theta}_{22}=\left[\begin{array}{cccc}
\Psi_{55} & 0 & 0 & \varepsilon E_{2}^{T} \\
* & \Psi_{66} & 0 & \varepsilon E_{3}^{T} \\
* & * & -\varepsilon I & 0 \\
* & * & * & -\varepsilon I
\end{array}\right],
\end{aligned}
$$

with $\Psi_{11}, \Psi_{22}, \Psi_{25}, \Psi_{55}, \Psi_{66}, L_{1}, L_{2}, X_{1}$, and $X_{2}$ defined in Theorem 7 .

Proof. We choose the same Lyapunov-Krasovskii functional as Theorem 7. According to neural network (2), we have the following equation:

$$
\begin{aligned}
r_{2}\left(t_{k}\right)=2 \delta^{T} x & \left(t_{k}\right) \\
\times S[ & -\left(W_{1}+\Delta W_{1}\left(t_{k}\right)\right) x\left(t_{k}\right) \\
& +\left(W_{2}+\Delta W_{2}\left(t_{k}\right)\right) f\left(x\left(t_{k}\right)\right) \\
& +\left(W_{3}+\Delta W_{3}\left(t_{k}\right)\right) f\left(x\left(t_{k}-d_{k}\right)\right) \\
& \left.-\delta x\left(t_{k}\right)\right]=0 .
\end{aligned}
$$

Similar to the proof of Theorem 7, we can have that

$$
\begin{aligned}
\delta V\left(t_{k}\right)= & \sum_{i=1}^{5} \delta V_{i}\left(t_{k}\right) \\
= & \sum_{i=1}^{5} \delta V_{i}\left(t_{k}\right)+r_{2}\left(t_{k}\right) \\
\leq & \eta^{T}\left(x\left(t_{k}\right)\right) \breve{\Xi} \eta\left(x\left(t_{k}\right)\right) \\
= & \eta^{T}\left(x\left(t_{k}\right)\right) \\
& \times\left(\Xi+\varphi F\left(t_{k}\right) \psi+\psi^{T} F^{T}\left(t_{k}\right) \varphi^{T}\right) \eta\left(x\left(t_{k}\right)\right),
\end{aligned}
$$


where

$$
\begin{aligned}
& \breve{\Xi}=\left[\begin{array}{cc}
\breve{\Theta}_{11} & \breve{\Theta}_{12} \\
* & \breve{\Theta}_{22}
\end{array}\right], \\
& \breve{\Theta}_{11}=\left[\begin{array}{ccc}
\Psi_{11} & -S W_{1 k}\left(t_{k}\right) & 0 \\
* & \breve{\Psi}_{22} & 0 \\
* & * & -Q_{2}
\end{array}\right] \text {, } \\
& \breve{\Theta}_{12}=\left[\begin{array}{ccc}
0 & S W_{2 k}\left(t_{k}\right) & S W_{3 k}\left(t_{k}\right) \\
\frac{1}{n_{M} T} R_{2} & P W_{2 k}\left(t_{k}\right) & \breve{\Psi}_{25} \\
0 & 0 & X_{2} K^{+}
\end{array}\right] \text {, } \\
& \breve{\Theta}_{22}=\left[\begin{array}{ccc}
-Q_{1}-\frac{1}{n_{M} T} R & 0 & 0 \\
* & \Psi_{55} & 0 \\
* & * & \Psi_{66}
\end{array}\right] \text {, } \\
& \varphi^{T}=\left[\begin{array}{llllll}
H^{T} S^{T} & H^{T} P^{T} & 0 & 0 & 0 & 0
\end{array}\right], \\
& \psi=\left[\begin{array}{llllll}
0 & -E_{1} & 0 & 0 & E_{2} & E_{3}
\end{array}\right], \\
& \breve{\Psi}_{22}=-2 L_{1} K^{-}+2 L_{2} K^{+}+Q_{1}+Q_{2} \\
& +T(d+1) Q_{2}-\frac{1}{n_{M} T} R \\
& -P W_{1 k}\left(t_{k}\right)-W_{1 k}^{T}\left(t_{k}\right) P, \\
& \breve{\Psi}_{25}=L_{1}-L_{2}+X_{1} K^{+}+P W_{2 k}\left(t_{k}\right) \text {, }
\end{aligned}
$$

with $W_{1 k}\left(t_{k}\right)=W_{1}+\Delta W_{1}\left(t_{k}\right), W_{2 k}\left(t_{k}\right)=W_{2}+\Delta W_{2}\left(t_{k}\right)$, $W_{3 k}\left(t_{k}\right)=W_{3}+\Delta W_{3}\left(t_{k}\right)$, and $\Xi, \Psi_{11}, \Psi_{55}, \Psi_{66}, L_{1}, L_{2}, X_{1}$, and $X_{2}$ have been defined in Theorem 7 .

Applying Schur complement to (30), we have

$$
\Xi+\varepsilon^{-1} \varphi \varphi^{T}+\varepsilon \psi^{T} \psi<0
$$

By Lemma 5, from the inequality (35), we can easily obtain

$$
\Xi+\varphi F\left(t_{k}\right) \psi+\psi^{T} F^{T}\left(t_{k}\right) \varphi^{T}<0
$$

Consequently, $\delta V\left(t_{k}\right)<0$; from Definition 1, neural network in (29) is robustly asymptotically stable. The proof is completed.

3.2. Passivity Analysis. In this subsection, the passivity analysis results are given in the following part. We first consider (2) and (3) without the parameter uncertainties $\Delta W_{i}\left(t_{k}\right)=0,(i=$ $1,2,3)$. Then, the following neural network can be obtained:

$$
\begin{aligned}
\delta x\left(t_{k}\right)= & -W_{1} x\left(t_{k}\right)+W_{2} f\left(x\left(t_{k}\right)\right) \\
& +W_{3} f\left(x\left(t_{k}-d_{k}\right)\right)+u\left(t_{k}\right), \\
& y\left(t_{k}\right)=f\left(x\left(t_{k}\right)\right) .
\end{aligned}
$$

Theorem 9. For given scalars $0 \leq d_{m} \leq d_{k} \leq d_{M}$, neural network is passive in (37), if there exist $P>0$,
$Q_{i}>0(i=1,2,3), R>0, S>0$ positive definite diagonal matrices $L_{1}, L_{2}, X_{1}$, and $X_{2}$, such that the following LMI holds:

$$
\widetilde{\Xi}<0,
$$

where

$$
\begin{aligned}
\widetilde{\Xi} & =\left[\begin{array}{cc}
\widetilde{\Theta}_{11} & \widetilde{\Theta}_{12} \\
* & \widetilde{\Theta}_{22}
\end{array}\right], \\
\widetilde{\Theta}_{12} & =\left[\begin{array}{cccc}
0 & S W_{2} & S W_{3} & S \\
\frac{1}{n_{M} T} R & \Psi_{25} & P W_{3} & P \\
0 & 0 & X_{2} K^{+} & 0
\end{array}\right], \\
\widetilde{\Theta}_{22} & =\left[\begin{array}{ccccc}
-Q_{1}-\frac{1}{n_{M} T} R & 0 & 0 & 0 \\
* & \Psi_{55} & 0 & -I \\
* & * & \Psi_{66} & 0 \\
* & * & * & -\gamma I
\end{array}\right],
\end{aligned}
$$

and $\widetilde{\Theta}_{11}=\Theta_{11}, \Psi_{25}, \Psi_{55}$, and $\Psi_{66}$ have been defined in Theorem 7 .

Proof. In order to present the passivity condition for neural network (37), we choose the same Lyapunov-Krasovskii functional as Theorem 7. By following the same line of proof of Theorem 7 and considering the following inequality:

$$
\begin{aligned}
\sum_{k=0}^{\infty}\left[-\gamma u^{T}\left(t_{k}\right) u\left(t_{k}\right)-2 y^{T}\left(t_{k}\right) u\left(t_{k}\right)\right] \\
=\sum_{k=0}^{\infty}\left[-\gamma u^{T}\left(t_{k}\right) u\left(t_{k}\right)-2 y^{T}\left(t_{k}\right) u\left(t_{k}\right)+\delta V\left(t_{k}\right)\right] \\
\quad-V(\infty)+V(0) \\
\leq \sum_{k=0}^{\infty}\left[-\gamma u^{T}\left(t_{k}\right) u\left(t_{k}\right)-2 y^{T}\left(t_{k}\right) u\left(t_{k}\right)+\delta V\left(t_{k}\right)\right],
\end{aligned}
$$

it can be seen from the LMI condition (40) that

$$
\sum_{k=0}^{\infty}\left[-\gamma u^{T}\left(t_{k}\right) u\left(t_{k}\right)-2 y^{T}\left(t_{k}\right) u\left(t_{k}\right)\right] \leq 0,
$$

which means that neural network in (37) is passive. This finishes the proof.

In the following theorem, the passivity condition for uncertain neural network with time-varying delay in (2) and (3) is presented.

Theorem 10. For given scalars $0 \leq d_{m} \leq d_{k} \leq d_{M}$, neural network in (2) and (3) is passive, if there exist $P>0, Q_{i}>$ 0 ( $i=1,2,3), R>0, S>0$ positive definite diagonal matrices $L_{1}, L_{2}, X_{1}$, and $X_{2}$, such that the following LMI holds:

$$
\left[\begin{array}{cc}
\bar{\Theta}_{11} & \bar{\Theta}_{12} \\
* & \bar{\Theta}_{22}
\end{array}\right]<0
$$


where

$$
\begin{gathered}
\bar{\Theta}_{12}=\left[\begin{array}{ccccc}
S W_{2} & S W_{3} & S & S H & 0 \\
\Psi_{25} & P W_{3} & P & P H & -\varepsilon E_{1}^{T} \\
0 & 0 & 0 & 0 & 0 \\
0 & X_{2} K^{+} & 0 & 0 & 0
\end{array}\right], \\
\bar{\Theta}_{22}=\left[\begin{array}{ccccc}
\Psi_{55} & 0 & -I & 0 & \varepsilon E_{2}^{T} \\
* & \Psi_{66} & 0 & 0 & \varepsilon E_{3}^{T} \\
* & * & -\gamma I & 0 & 0 \\
* & * & * & -\varepsilon I & 0 \\
* & * & * & * & -\varepsilon I
\end{array}\right]
\end{gathered}
$$

and $\bar{\Theta}_{11}=\widehat{\Theta}_{11}, \Psi_{25}, \Psi_{55}$, and $\Psi_{66}$ have been defined in Theorem 8 .

Proof. In order to present the passivity condition for neural network in (2) and (3), we choose the same LyapunovKrasovskii functional as Theorem 7. By following the same line of proof of Theorems 8 and 9, Theorem 10 can be proved.

\section{A Numerical Example}

In this section, the following numerical example is presented to demonstrate the effectiveness of the proposed results.

Example 1. Consider discrete-time neural networks (2) and (3) with the following parameters:

$$
\begin{aligned}
& W_{1}=\left[\begin{array}{ll}
2 & 0 \\
0 & 2
\end{array}\right], \quad W_{2}=\left[\begin{array}{cc}
1 & 1 \\
-1 & -1
\end{array}\right], \\
& W_{3}=\left[\begin{array}{cc}
0.88 & 1 \\
1 & 1
\end{array}\right], \\
& H=0.1 \times I, \quad E_{1}=0.1 \times I, \\
& E_{2}=0.2 \times I, \quad E_{3}=0.3 \times I, \\
& K^{-}=\operatorname{diag}\{-1,-1\}, \\
& K^{+}=\operatorname{diag}\{0.9,0.9\} .
\end{aligned}
$$

In order to illustrate the effectiveness of the obtained results, we choose $T=0.05, n_{m}=10$, and $n_{M}=20$. Then, using the Matlab LMI toolbox to solve the LMI in (42), we obtain a solution as follows:

$$
\begin{aligned}
P & =\left[\begin{array}{ll}
5.2080 & 2.0984 \\
2.0984 & 9.7576
\end{array}\right], \\
S & =\left[\begin{array}{ll}
1.2475 & 0.1776 \\
0.1776 & 2.9572
\end{array}\right], \\
Q_{1} & =\left[\begin{array}{cc}
0.2133 & -0.0219 \\
-0.0219 & 1.2999
\end{array}\right], \\
Q_{2} & =\left[\begin{array}{ll}
0.4684 & 0.2752 \\
0.2752 & 1.7084
\end{array}\right], \\
Q_{3} & =\left[\begin{array}{ll}
7.3290 & 6.0654 \\
6.0654 & 12.4586
\end{array}\right],
\end{aligned}
$$

$$
\begin{aligned}
& R=\left[\begin{array}{ll}
0.6211 & 0.0621 \\
0.0621 & 1.5979
\end{array}\right] \\
& L_{1}=\left[\begin{array}{cc}
0.0313 & 0 \\
0 & 0.2507
\end{array}\right] \text {, } \\
& L_{2}=\left[\begin{array}{cc}
2.7406 & 0 \\
0 & 0.7734
\end{array}\right] \text {, } \\
& X_{1}=\left[\begin{array}{cc}
9.4819 & 0 \\
0 & 31.8737
\end{array}\right] \text {, } \\
& X_{2}=\left[\begin{array}{cc}
0.6547 & 0 \\
0 & 3.2283
\end{array}\right] \text {, } \\
& \gamma=48.7332, \quad \varepsilon=3.1900 \text {. }
\end{aligned}
$$

\section{Conclusions}

In this paper, the problems of stability and passivity analysis for discrete-time neural networks with time-varying delay have been studied via delta operator approach. This paper has considered the parameter uncertainty and the generalized activation functions. By constructing appropriate LyapunovKrasovskii functional, some novel stability and passivity criteria have been proposed in the delta operator system framework. The obtained conditions have been expressed in terms of LMI, which can be easily solved by standard software. A numerical example has been given to illustrate the effectiveness of the proposed results.

\section{Conflict of Interests}

The authors declare that there is no conflict of interests regarding the publication of this paper.

\section{Acknowledgment}

This work was partially supported by the National Natural Science Foundation of China (nos. 61304003 and 11226138).

\section{References}

[1] G. A. Carpenter, "Neural network models for pattern recognition and associative memory," Neural Networks, vol. 2, no. 4, pp. 243-257, 1989.

[2] A. N. Michel, J. A. Farrell, and H.-F. Sun, "Analysis and synthesis techniques for Hopfield type synchronous discrete time neural networks with application to associative memory," Institute of Electrical and Electronics Engineers. Transactions on Circuits and Systems, vol. 37, no. 11, pp. 1356-1366, 1990.

[3] M. Galicki, H. Witte, J. Dörschel, M. Eiselt, and G. Griessbach, "Common optimization of adaptive preprocessing units and a neural network during the learning period. Application in EEG pattern recognition," Neural Networks, vol. 10, no. 6, pp. 11531163, 1997.

[4] Y. Hayakawa, A. Marumoto, and Y. Sawada, "Effects of the chaotic noise on the performance of a neural network model 
for optimization problems," Physical Review E, vol. 51, no. 4, pp. R2693-R2696, 1995.

[5] C. Peterson and B. Soderberg, "A new method for mapping optimization problems onto neural networks," International Journal of Neural Systems, vol. 1, no. 01, pp. 3-22, 1989.

[6] S. Yin, H. Luo, and S. X. Ding, "Real-time implementation of faulttolerant control systems with performance optimization," IEEE Transactions on Industrial Electronics, vol. 61, no. 5, pp. 2402-2411, 2013.

[7] S. Yin, S. X. Ding, A. Haghani, H. Hao, and P. Zhang, "A comparison study of basic data-driven fault diagnosis and process monitoring methods on the benchmark tennessee eastman process," Journal of Process Control, vol. 22, no. 9, pp. 1567-1581, 2012.

[8] J. Cao, D.-S. Huang, and Y. Qu, "Global robust stability of delayed recurrent neural networks," Chaos, Solitons and Fractals, vol. 23, no. 1, pp. 221-229, 2005.

[9] R. Yang, Z. Zhang, and P. Shi, "Exponential stability on stochastic neural networks with discrete interval and distributed delays," IEEE Transactions on Neural Networks, vol. 21, no. 1, pp. 169-175, 2010.

[10] T. Chen and L. Rong, "Delay-independent stability analysis of Cohen-Grossberg neural networks," Physics Letters A, vol. 317, no. 5-6, pp. 436-449, 2003.

[11] S. Arik, "An analysis of global asymptotic stability of delayed cellular neural networks," IEEE Transactions on Neural Networks, vol. 13, no. 5, pp. 1239-1242, 2002.

[12] H. Li, B. Chen, Q. Zhou, and W. Qian, "Robust stability for uncertain delayed fuzzy Hopfield neural networks with Markovian jumping parameters," IEEE Transactions on Systems, Man, and Cybernetics B, vol. 39, no. 1, pp. 94-102, 2009.

[13] C. Li and X. Liao, "Passivity analysis of neural networks with time delay," IEEE Transactions on Circuits and Systems II, vol. 52, no. 8, pp. 471-475, 2005.

[14] S. Xu, W. X. Zheng, and Y. Zou, "Passivity analysis of neural networks with time-varying delays," IEEE Transactions on Circuits and Systems II, vol. 56, no. 4, pp. 325-329, 2009.

[15] Q. Song, J. Liang, and Z. Wang, "Passivity analysis of discretetime stochastic neural networks with time-varying delays," Neurocomputing, vol. 72, no. 7-9, pp. 1782-1788, 2009.

[16] X. Li and H. Gao, "A new model transformation of discrete-time systems with time-varying delay and its application to stability analysis," Institute of Electrical and Electronics Engineers. Transactions on Automatic Control, vol. 56, no. 9, pp. 2172-2178, 2011.

[17] H. Gao and X. Li, " $H_{\infty}$ filtering for discrete-time statedelayed systems with finite frequency specifications," Institute of Electrical and Electronics Engineers. Transactions on Automatic Control, vol. 56, no. 12, pp. 2935-2941, 2011.

[18] H. Li, H. Liu, H. Gao, and P. Shi, "Reliable fuzzy control for active suspension systems with actuator delay and fault," IEEE Transactions on Fuzzy Systems, vol. 20, no. 2, pp. 342-357, 2012.

[19] H. Li, X. Jing, and H. R. Karimi, "Output-feedback based $H_{\infty}$ control for active suspension systems with control delay," IEEE Transactions on Industrial Electronics, vol. 61, no. 1, pp. 436-446, 2014.

[20] L. Wu and W. X. Zheng, "Passivity-based sliding mode control of uncertain singular time-delay systems," Automatica, vol. 45, no. 9, pp. 2120-2127, 2009.

[21] J. Qiu, G. Feng, and J. Yang, "A new design of delay-dependent robust $H_{\infty}$ filtering for discrete-time T-S fuzzy systems with time-varying delay," IEEE Transactions on Fuzzy Systems, vol. 17, no. 5, pp. 1044-1058, 2009.
[22] L. Wu and W. X. Zheng, "Weighted $H_{\infty}$ model reduction for linear switched systems with time-varying delay," Automatica, vol. 45, no. 1, pp. 186-193, 2009.

[23] J. Qiu, G. Feng, and J. Yang, "Improved delay-dependent $H_{\infty}$ filtering design for discrete-time polytopic linear delay systems," IEEE Transactions on Circuits and Systems II, vol. 55, no. 2, pp. 178-182, 2008.

[24] H. Gao, T. Chen, and J. Lam, "A new delay system approach to network-based control," Automatica, vol. 44, no. 1, pp. 39-52, 2008.

[25] H. Gao and T. Chen, "New results on stability of discrete-time systems with time-varying state delay," Institute of Electrical and Electronics Engineers. Transactions on Automatic Control, vol. 52, no. 2, pp. 328-334, 2007.

[26] M. Liu, P. Shi, L. Zhang, and X. Zhao, "Fault-tolerant control for nonlinear Markovian jump systems via proportional and derivative sliding mode observer technique," IEEE Transactions on Circuits and Systems. I, vol. 58, no. 11, pp. 2755-2764, 2011.

[27] S. Tong and Y. Li, "Adaptive fuzzy output feedback tracking backstepping control of strict-feedback nonlinear systems with unknown dead zones," IEEE Transactions on Fuzzy Systems, vol. 20, no. 1, pp. 168-180, 2012.

[28] S. Tong, Y. Li, Y. Li, and Y. Liu, "Observer-based adaptive fuzzy backstepping control for a class of stochastic nonlinear strictfeedback systems," IEEE Transactions on Systems, Man, and Cybernetics B, vol. 41, no. 6, pp. 1693-1704, 2011.

[29] S. Tong, Y. Liu, and T. Li, "Adaptive fuzzy robust output feedback control of nonlinear systems with unknown dead zones based on small-gain approach," IEEE Transactions on Fuzzy Systems, vol. 22, no. 1, pp. 164-176, 2014.

[30] S. Xu, J. Lam, D. W. C. Ho, and Y. Zou, "Delay-dependent exponential stability for a class of neural networks with time delays," Journal of Computational and Applied Mathematics, vol. 183, no. 1, pp. 16-28, 2005.

[31] Z. Wang, Y. Liu, M. Li, and X. Liu, "Stability analysis for stochastic Cohen-Grossberg neural networks with mixed time delays," IEEE Transactions on Neural Networks, vol. 17, no. 3, pp. 814-820, 2006.

[32] C. Hua, C. Long, and X. Guan, "New results on stability analysis of neural networks with time-varying delays," Physics Letters A, vol. 352, no. 4-5, pp. 335-340, 2006.

[33] S. Xu and J. Lam, "A new approach to exponential stability analysis of neural networks with time-varying delays," Neural Networks, vol. 19, no. 1, pp. 76-83, 2006.

[34] Y. He, G. P. Liu, D. Rees, and M. Wu, "Stability analysis for neural networks with time-varying interval delay," IEEE Transactions on Neural Networks, vol. 18, no. 6, pp. 1850-1854, 2007.

[35] S. Arik, "Further analysis of stability of uncertain neural networks with multiple time delays," Advances in Difference Equations, vol. 2014, article 41, 2014.

[36] Z. Orman and S. Arik, "An analysis of stability of a class of neutral-type neural networks with discrete time delays," Abstract and Applied Analysis, vol. 2013, Article ID 143585, 9 pages, 2013.

[37] Z.-G. Wu, P. Shi, H. Su, and J. Chu, "Passivity analysis for discrete-time stochastic markovian jump neural networks with mixed time delays," IEEE Transactions on Neural Networks, vol. 22, no. 10, pp. 1566-1575, 2011.

[38] H. Li, C. Wang, P. Shi, and H. Gao, "New passivity results for uncertain discrete-time stochastic neural networks with mixed time delays," Neurocomputing, vol. 73, no. 16-18, pp. 3291-3299, 2010. 
[39] H. Li, H. Gao, and P. Shi, "New passivity analysis for neural networks with discrete and distributed delays," IEEE Transactions on Neural Networks, vol. 21, no. 11, pp. 1842-1847, 2010.

[40] D. H. Ji, J. H. Koo, S. C. Won, S. M. Lee, and J. H. Park, "Passivity-based control for Hopfield neural networks using convex representation," Applied Mathematics and Computation, vol. 217, no. 13, pp. 6168-6175, 2011.

[41] A. Wu and Z. Zeng, "Exponential passivity of memristive neural networks with time delays," Neural Networks, vol. 49, pp. 11-18, 2014.

[42] G. C. Goodwin, R. L. Leal, D. Q. Mayne, and R. H. Middleton, "Rapprochement between continuous and discrete model reference adaptive control," Automatica, vol. 22, no. 2, pp. 199-207, 1986.

[43] C. B. Soh, "Robust stability of discrete-time systems using delta operators," Institute of Electrical and Electronics Engineers. Transactions on Automatic Control, vol. 36, no. 3, pp. 377-380, 1991.

[44] Y. Xia, M. Fu, H. Yang, and G.-P. Liu, "Robust sliding-mode control for uncertain time-delay systems based on delta operator," IEEE Transactions on Industrial Electronics, vol. 56, no. 9, pp. 3646-3655, 2009.

[45] J. Qiu, Y. Xia, H. Yang, and J. Zhang, "Robust stabilisation for a class of discrete-time systems with time-varying delays via delta operators," IET Control Theory \& Applications, vol. 2, no. 1, pp. 87-93, 2008.

[46] S. Li, Z. Xiang, and H. R. Karimi, "Finite-time $L_{1}$-gain control for positive switched systems with time-varying delay via delta operator approach," Abstract and Applied Analysis, vol. 2014, Article ID 872158, 11 pages, 2014.

[47] X. Xie, S. Yin, H. Gao, and O. Kaynak, "Asymptotic stability and stabilisation of uncertain delta operator systems with timevarying delays," IET Control Theory \& Applications, vol. 7, no. 8, pp. 1071-1078, 2013.

[48] H. Yang, Y. Xia, M. Fu, and P. Shi, "Robust adaptive sliding mode control for uncertain delta operator systems," International Journal of Adaptive Control and Signal Processing, vol. 24, no. 8, pp. 623-632, 2010.

[49] Z. R. Xiang, Q. W. Chen, W. L. Hu, and D. J. Zhang, "Robust stability analysis and control for fuzzy systems with uncertainties using the delta operator," Control and Decision, vol. 18, no. 6, pp. 720-723, 2003.

[50] X. Jiang, Q.-L. Han, and X. Yu, "Stability criteria for linear discrete-time systems with interval-like time-varying delay," in Proceedings of the American Control Conference (ACC '05), pp. 2817-2822, June 2005.

[51] M. S. Mahmoud, Resilient Control of Uncertain Dynamical Systems, vol. 303 of Lecture Notes in Control and Information Sciences, Springer, Berlin, Germany, 2004.

[52] S. Boyd, L. El Ghaoui, E. Feron, and V. Balakrishnan, Linear Matrix Inequalities in System and Control Theory, vol. 15 of SIAM Studies in Applied Mathematics, Society for Industrial and Applied Mathematics (SIAM), Philadelphia, Pa, USA, 1994. 


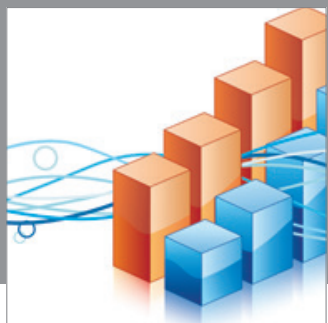

Advances in

Operations Research

mansans

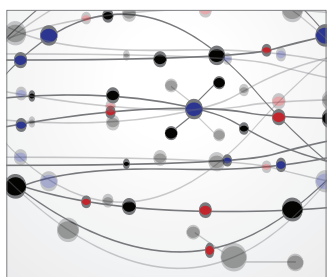

The Scientific World Journal
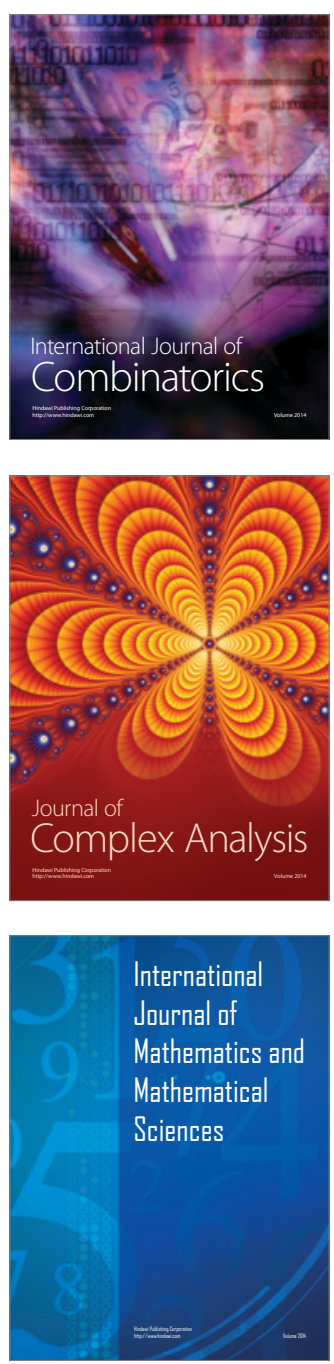
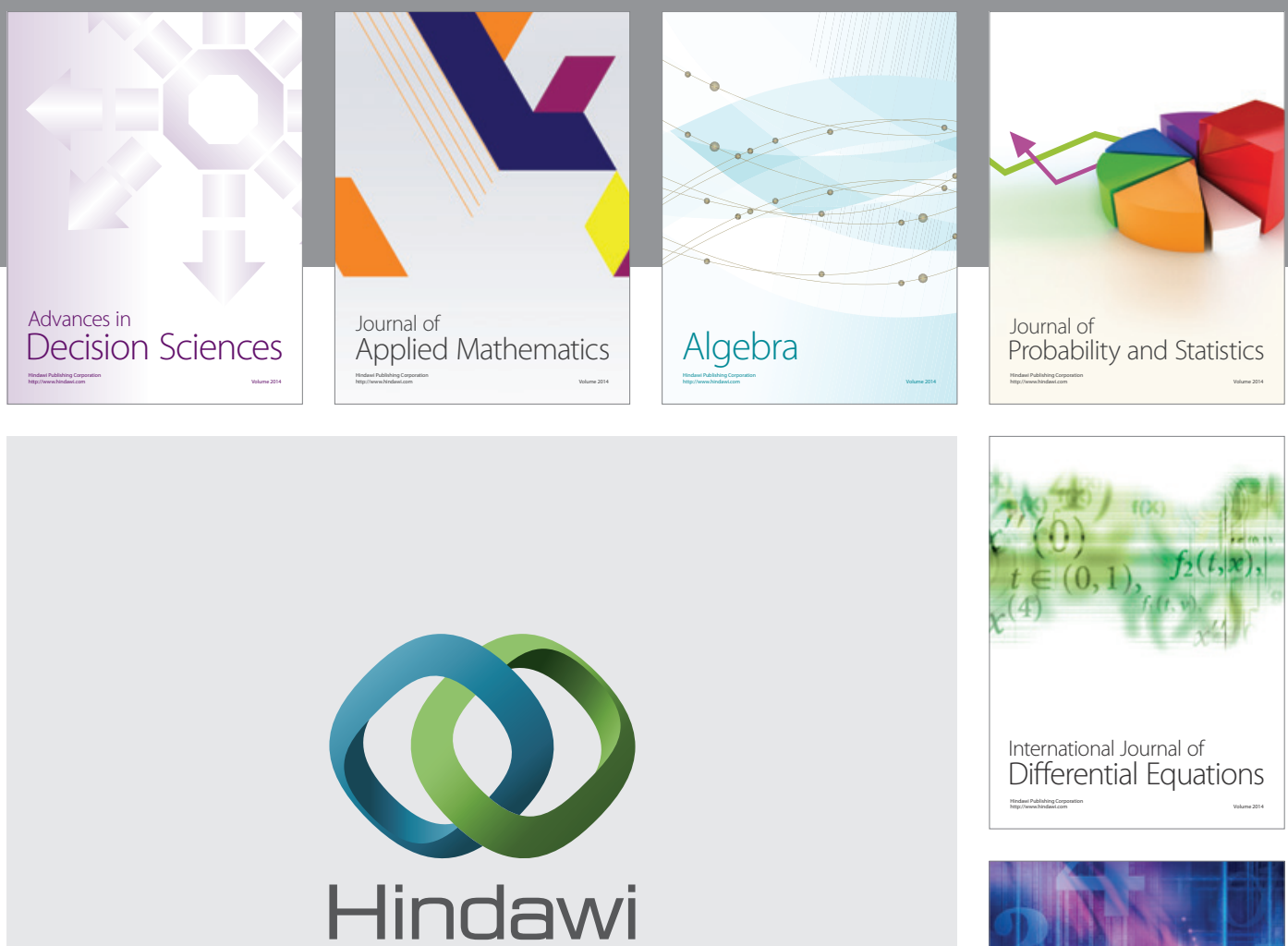

Submit your manuscripts at http://www.hindawi.com
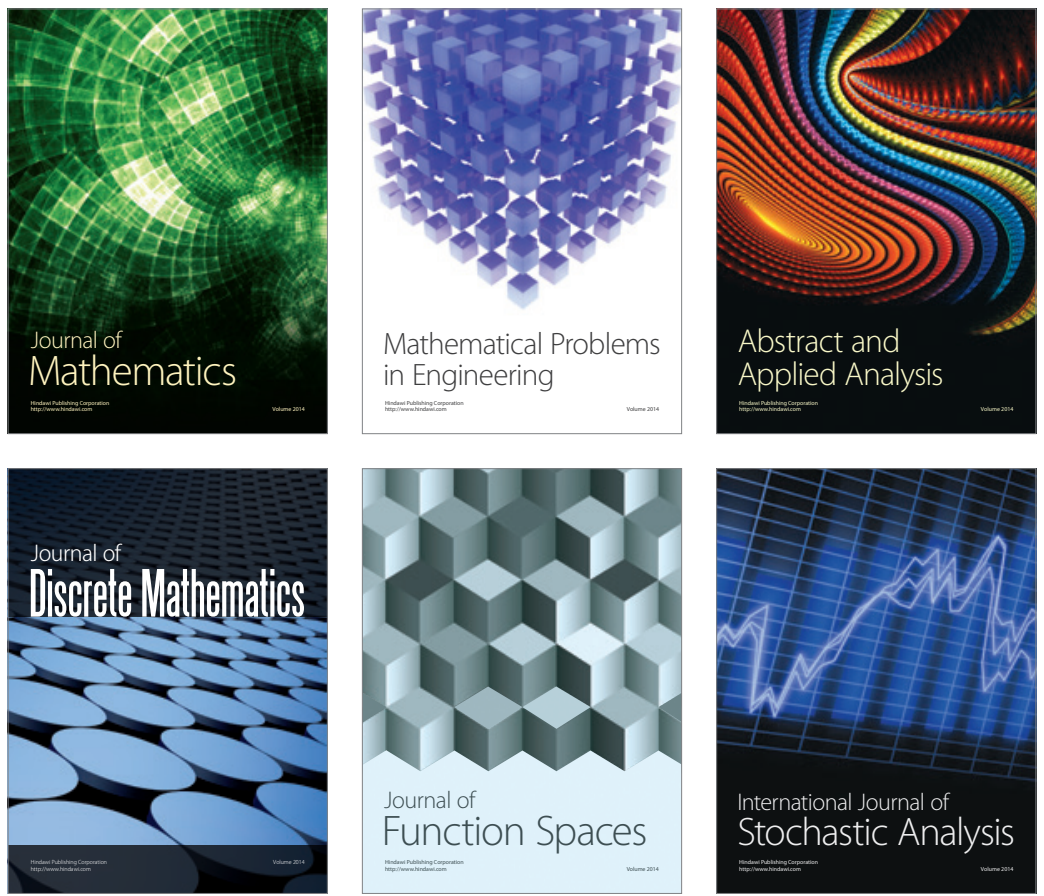

Journal of

Function Spaces

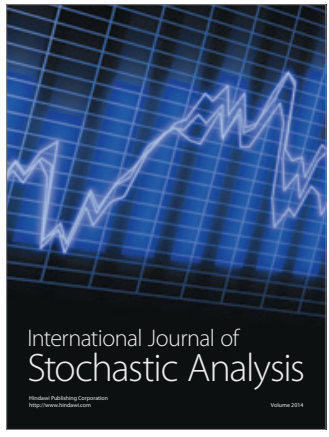

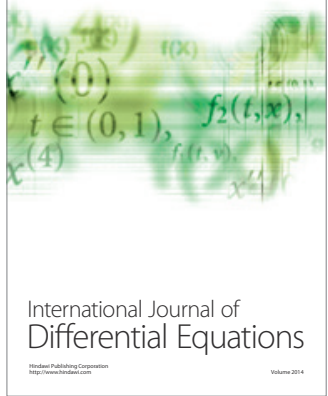
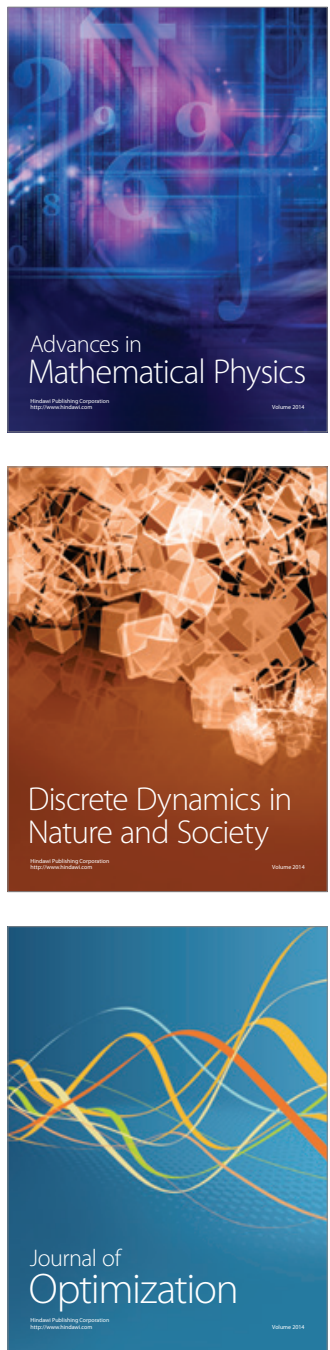